\title{
Laboreal
}

Volume $3 \mathrm{~N}^{0} 1$ | 2007

Varia

\section{Aspectos funcionales de los riesgos y desviaciones de las normas de seguridad en el trabajo : una aportación para la comprensión de las relaciones entre actividad humana y seguridad}

Aspectos funcionais dos riscos e desvios das normas de segurança no trabalho: um contributo para a compreensão das relações entre actividade humana e segurança

Aspects fonctionnels des risques et écarts par rapport aux normes de sécurité au travail : une contribution pour la compréhension des relations entre activité humaine et sécurité

Functional aspects of hazards and safety at work rules' deviations: a contribution to the understanding of the relations between human activity and safety

\section{Mário Poy}

\section{OpenEdition}

\section{Journals}

Edición electrónica

URL: http://journals.openedition.org/laboreal/12946

DOI: $10.4000 /$ laboreal.12946

ISSN: 1646-5237

\section{Editor}

Universidade do Porto

\section{Referencia electrónica}

Mário Poy, «Aspectos funcionales de los riesgos y desviaciones de las normas de seguridad en el trabajo : una aportación para la comprensión de las relaciones entre actividad humana y seguridad », Laboreal [En línea], Volume $3 N^{0} 1 \mid$ 2007, Publicado el 01 julio 2007, consultado el 24 septiembre 2020. URL : http://journals.openedition.org/laboreal/12946; DOI : https://doi.org/10.4000/laboreal.12946

Este documento fue generado automáticamente el 24 septiembre 2020

Laboreal está licenciado com uma Licença Creative Commons - Atribuição-NãoComercial 4.0 Internacional. 


\section{Aspectos funcionales de los riesgos y desviaciones de las normas de seguridad en el trabajo : una aportación para la comprensión de las relaciones entre actividad humana y seguridad}

Aspectos funcionais dos riscos e desvios das normas de segurança no trabalho: um contributo para a compreensão das relações entre actividade humana e segurança

Aspects fonctionnels des risques et écarts par rapport aux normes de sécurité au travail : une contribution pour la compréhension des relations entre activité humaine et sécurité

Functional aspects of hazards and safety at work rules' deviations : a contribution to the understanding of the relations between human activity and safety

Mário Poy

\section{REFERENCIA}

Poy, M. (2007). Aspectos funcionales de los riesgos y desvios de las normas de seguridad en el trabajo. Un aporte a la comprensión de las relaciones entre actividad humana y seguridad. Tese de Doutoramento, Universidade de Palermo, Buenos Aires. 


\section{NOTA DEL EDITOR}

Manuscrito recibido en : marzo/2007

Aceptado tras peritage en : junio/2007

\section{El contexto social y científico de esta indagación}

1 Durante la década de los años 90, hemos asistido en Argentina a una serie de importantes transformaciones en la economía y en los sectores de la producción, que han afectado en forma significativa al trabajo humano y a la seguridad en general [1]. Entre otras, junto al proceso de privatización de las empresas públicas, se han sucedido cambios significativos en las formas de producción, en la organización del trabajo y en las relaciones laborales. Es así que a la introducción de nuevas tecnologías informatización y automatización de tareasse le han sumado modalidades de contratación laboral precarizadas, la tercerización -sobre todo de las tareas de riesgo-, la incorporación de modelos de gestión tales como el just in time, los círculos de calidad, el trabajo en células, y otras formas de organización del trabajo que, transferidas desde otros contextos culturales y económicos, han modificado sustancialmente los procesos de trabajo y el empleo.

2 En nuestro país, la seguridad en el trabajo viene planteando desde hace muchos años una serie de dificultades, cuyos alcances se hacen sentir tanto en el plano social como en el económico. A título ilustrativo, las estadísticas sobre empleo formal [ [2] (aproximadamente 6 millones de personas asalariadas) muestran que se registra una mortalidad de 155 por millón; una incidencia anual de accidentes y enfermedades profesionales que alcanza a 80 de cada 1000 trabajadores-año, y una pérdida de productividad de 7.000 .000 días-año.

3 Por otra parte, el estudio de las relaciones entre salud y trabajo, y más particularmente los problemas referidos al campo de la seguridad y la confiabilidad humana, aparecen, dentro del contexto de nuestro país, escasamente abordados por la psicología del trabajo y la ergonomía. Es así que, de forma más o menos explícita, los enfoques psicológicos adoptados cuando se trata de explicar los accidentes de trabajo, independientemente de su nivel de complejidad, tienden a centrar las causas en las personas que se encuentran en las primeras líneas de los sistemas de trabajo, en forma casi excluyente. En consecuencia, por ejemplo, la toma de riesgos y los desvíos deliberados de las normas de seguridad, objetos de nuestra investigación, son raramente vistos como síntomas de disfuncionamiento dentro de un sistema determinado, tal como lo preconizan algunos enfoques más contemporáneos (Reason, 1993 ; Rasmussen, 1997 ; Leplat, 1997), sino más bien como elementos individuales que deben ser eliminados.

4 Esta falta de atención sistemática hace que, con frecuencia, en el plano de las prácticas profesionales concretas, las interpretaciones sobre el accionar de las personas queden libradas a supuestos psicológicos que muchas veces no están empíricamente validados. 


\section{Las relaciones salud-seguridad en operadores del sector sidero-metalúrgico : objetivos y metodología del estudio}

\subsection{Objetivos}

En este trabajo nos proponemos indagar cuáles son las concepciones que poseen los "no expertos" [3] (Weill Fassina, Kouabenan \& De La Garza, 2005) acerca de los riesgos y de los desvíos de las normas de seguridad en el trabajo, y cómo dichas representaciones evolucionan y varían, según se refieran a los aspectos prescritos o reales del trabajo, a la formación, a la edad y/o al tamaño de empresa.

Esta investigación llevada a cabo en Argentina, en el sector sidero-metalúrgico, a partir de una muestra intencional de 133 operadores y 43 operadores/delegados pertenecientes a una Gran empresa y a un conglomerado de 16 Pequeñas y Medianas Empresas (PyMES), nos ha posibilitado poner de manifiesto el grado de reconocimiento de la toma de riesgos y de los desvíos de las normas de seguridad, así como comprender las razones asociadas a dichas acciones.

7 Nuestra perspectiva teórica, de carácter ecléctica, centrada en los enfoques de la ingeniería cognitiva (Rasmussen, 1997), la psicología ergonómica (Leplat, 1997), y la clínica de la actividad (Clot, 1999), nos ha permitido generar una malla de análisis con la que capturar y enriquecer los resultados obtenidos.

\subsection{Metodología empleada en el estudio}

8 A fin de dotar de mayor consistencia a nuestros datos, hemos creído necesario conjugar los enfoques metodológicos cuantitativos y cualitativos. Este cruce deliberado de perspectivas teóricas y de métodos nos ha permitido abordar las relaciones hombretrabajo-seguridad, de modo tal de poder aportar algunos resultados que permitan el enriquecimiento o la construcción de nuevos modelos de compresión (Vaxevanoglou \& Ponnelle, 1999).

9 En un plano instrumental, hemos adaptado una encuesta (Boix \& al., 2001) al contexto local y sectorial, administrándola a la población de los operadores.

10 Asimismo, hemos elaborado una guía de entrevistas dirigida a los operadores/ delegados, con el objetivo de obtener mayor riqueza de información.

\section{Toma de riesgos y desvíos de las normas de seguridad : características comunes y rasgos específicos}

11 A continuación, presentamos los resultados referidos a la representación de los riesgos y los desvíos para la población en general (3.1.), para luego poner en consideración el papel jugado por la edad y el tamaño de empresa (3.2.) y, finalmente, sintetizar la concepción de los operadores/delegados. 


\subsection{Los riesgos y los desvíos : prácticas frecuentes en el trabajo}

12 Consultados sobre la frecuencia y el grado de toma de riesgo, los resultados muestran que el $83 \%$ operadores sostiene que, con diferente frecuencia, se arriesgan mucho en el trabajo. El $30 \%$ declara hacerlo siempre o casi siempre.

13 Entre las razones asociadas a los riesgos, la capacitación pareciera no ser una herramienta tan efectiva como en un principio sostenían los operadores: el $63 \%$ reconoce déficit en la adecuación entre capacitación y seguridad.

14 A su vez, el $45 \%$ de las opiniones dejan entrever que no hay un control adecuado por parte de la supervisión.

15 Asimismo, los puntos de conflicto revelados entre, por un lado, la seguridad y el ritmo de trabajo y, por otro lado, la seguridad y la productividad no hacen sino revelar ciertos desfasajes entre los procedimientos y la realidad del trabajo, que justificarían asumir riesgos y desviarse de las normas de seguridad. Es así que aproximadamente el $65 \%$ de los operarios declara que trabajar conforme a normas implica no alcanzar los objetivos de la producción.

16 Con relación al reconocimiento explícito de trabajar desviándose de la normas de seguridad el $73 \%$ de los operadores sostiene, con diferentes grados de frecuencia, desviarse de las mismas

17 Entre las razones que evocan en cuanto al desvío en el cumplimiento de las normas, señalan la dificultad en la realización de las tareas, preferir sus propios métodos de seguridad, y que el cumplimento de las mismas les impide alcanzar los objetivos de la producción.

18 Estos desvíos parecen entonces obedecer a un doble cuestión : por un lado, a una inadecuación o falta de acoplamiento entre las normas que rigen a la producción y las que rigen a la seguridad y, por otro lado, a una falta de mecanismos que permitan la actualización o, incluso, la creación de normas más adaptadas al contexto de las empresas.

\subsection{La edad y el tamaño de empresa}

19 Si se tiene en cuenta la edad respecto de la toma de riesgos y desvíos de las normas de seguridad, resultan ser los operadores más jóvenes quienes dicen arriesgarse mucho en el trabajo (90\%). Las razones varían con la edad: los primeros parecen verse más afectados por la falta o la inadecuación de la formación, mientras que los de mayor edad asocian mayormente los riesgos a la imposibilidad de cumplir con los objetivos de la producción.

20 Respecto del desvío deliberado de las normas de seguridad, son los más jóvenes quienes explícitamente reconocen desviarse (siempre o casi siempre), mientras que los de más edad se sitúan proporcionalmente en el otro extremo: dicen hacerlo sólo a veces o nunca.

21 Respecto de las razones asociadas a los desvíos, para los más jóvenes es la preferencia por sus propios métodos de prevención, y en los de más edad es porque hacen más difíciles las tareas. 

operadores PyMES, porque prefieren sus propios métodos de seguridad y, para los operadores de la Gran empresa, porque las normas dificultan sus tareas. Estas razones dejan suponer que la inadecuación entre las normas de seguridad y la realidad del trabajo es más marcada en el sector PyMES, pero, al mismo tiempo, también sugiere quizás un mayor margen de discrecionalidad (Maggi, 2003) en el trabajo. En este sentido, podemos suponer que el alejarse de las normas no es sólo una trasgresión, o que ésta no es necesariamente negativa, tal como lo muestran los trabajos de Rabardel (1995).

Finalmente, en el análisis fino del discurso de los delegados/ operarios, hemos encontrado resultados que apuntalan a los anteriores. Esta forma de proceder nos ha permitido confirmar que las fuentes de adquisición de los conocimientos en materia de seguridad son más bien producto del trabajo "en el puesto", que de las capacitaciones formales impartidas. Asimismo, los desvíos de las normas, las razones asociadas, son imputadas a la falta de acoplamiento entre seguridad y productividad. El tiempo, en todas sus dimensiones : ritmo, cadencia, superposición de tareas, aparece como la razón más evocada por los "no expertos".

Pareciera no existir un referencial común respecto de quiénes y de cómo se construyen las normas y procedimientos en el trabajo, que regulan, la acción de los operarios. Esta cuestión refuerza, entonces, la idea de que lejos de carecer de normas, existirían unas normas instrumentales (Rabardel, op.cit.) y una regulación de carácter más bien implícito, que estaría definida por el género profesional (Clot, 1999).

\section{Conclusiones y vías de reflexión posibles}

Los resultados presentados muestran que la toma de riesgos y los desvíos de las normas de seguridad son acciones ampliamente reconocidas y aceptadas por los operadores, dentro del contexto de sus trabajos. Parece tratarse de una forma de regulación que intenta, de forma más o menos exitosa, saldar los conflictos resultantes de dos lógicas a menudo contradictorias : la de la producción y la de la seguridad. En este sentido, nuestro estudio sugiere que la toma de riesgos y el desvío de las normas de seguridad no son, per se, necesariamente negativos, reforzando la idea de que, finalmente, no se trata solamente de eliminarlos sino, más bien, de generar dispositivos en la organización que permitan su formalización y posibiliten su gestión. Es decir, poder identificar los factores que los determinan, a fin de favorecer acciones que permitan 
tanto la modificación eventual de las normas de seguridad, como las correcciones de las consignas de producción o la organización del trabajo.

La formación, la edad y el tamaño de la empresa, parecen ser elementos que modulan e imprimen características específicas a ambas formas de acción.

El rol de los aprendizajes informales, más o menos conscientes, construidos en el trabajo y que permiten una gestión de los riesgos, nos lleva a interrogarnos acerca del grado de reconocimiento formal de los mismos por parte de la organización del trabajo, así como acerca del grado de acoplamiento entre éstos y los contenidos de las capacitaciones formales impartidas.

1 Por otro lado, la edad demuestra ser un elemento importante, en el sentido de la construcción de estrategias más eficientes en la gestión de los riesgos. En este sentido, los resultados de esta investigación van en la misma dirección de los de Pueyo (1999), llevando a cuestionar los modelos de tipo "deficitarios" respecto de la edad, en los cuales son sólo los jóvenes quienes podrían adaptarse a las exigencias de los sistemas productivos.

32 Asimismo, el tamaño de la empresa pareciera jugar un rol distintivo, cuenta habida de las diferencias relevadas en cuanto a la toma de riesgos y a los desvíos de las normas de seguridad, y a las razones asociadas.

Concluyendo, debemos señalar que nuestro estudio no ha podido profundizar en la dinámica de funcionamiento de los desvíos, ni en la multiplicidad de razones que operan como motores que empujan al sistema hacia la zona donde los accidentes son posibles (Amalberti, 2001). En este aspecto, sería deseable indagar acerca de los mecanismos que nos permitan dar cuenta de las formas de negociación de los límites a las migraciones de las normas de seguridad, por parte de los colectivos de trabajo.

En otras palabras, sería deseable poder describir con mayor precisión cuáles son las zonas de tolerancia y cuáles no lo son, intentando describir, al mismo tiempo, las interrelaciones entre las acciones individuales y la dimensión colectiva de la acción. En este sentido, los estudios sobre situaciones normales deberían multiplicarse, a fin de poder capturar no solamente los mecanismos que conducen a un accidente, sino también los que permiten evitarlo.

\section{BIBLIOGRAFÍA}

Amalberti, R. (2001). The paradoxes of almost totally safe transportation systems. Safety Science $37,2 / 3,109-126$.

Boix, P., Torada, R. \& alt. (2001). Necesidades de formación y percepción de riesgos laborales de los trabajodores/as en el sector de fabricación de azulejos, pavimentos y baldosas cerámicas. Valencia : ISTAS.

Clot, Y. (1999). La fonction psychologique du travail. Paris : Presses Universitaires de France. 
Hoc, J. M. \& Darses, F. (2004). Introduction. In Hoc, J. M. \& Darses, F. Eds. Psychologie ergonomique : tendances actuelles (pp. 1-13). Paris. Presses Universitaries de France.

Leplat, J. (1997). Regards sur l'activité en situation de travail - Contribution à la psychologie ergonomique. Paris : Presses Universitaires de France.

Maggi, B. (2003). De l'agir organisationnel. Un point de vue sur le travail, le bien-être, 1 'apprentissage. Collection Travail \& activité humaine. Toulouse : Octarès Editions.

Pueyo, V. (1999). Régulation de l'efficience avec l'expérience : quelles questions pour la construction d'indicateurs de suivi de la production? XXXIVe Congrès de la SELF, pp. 221-230. Caen, France.

Rabardel, P. (1995.) Les Hommes et les Technologies. Approche cognitive des instruments contemporains. Paris : Armand Collin.

Rasmussen, J. (1997). Risk management in a dynamic society : a modelling problem. Safety Science, 27, 2/3, 183-213.

Reason, J. (1993). L'erreur humaine. Paris : Presses Universitaires de France. (Translated by J.M. Hoc, Human error, 1990, Cambridge University Press).

Vaxevanoglou, X. \& Ponnelle, S. (Septembre, 1999). Ajustement au stress et santé au travail. Communication presentée au 34 congrés de la SELF. Caen, France.

Weill Fassina, A, Kouabenan, R. \& De La Garza, C. (2005). Analyse des accidents du travail, gestion des risques et prévention. Manuel de Psychologie des Organisations. Grenoble : Kouabenan, R. Presses Universitaires de Grenoble.

\section{NOTAS}

1. Los dos accidentes de la aviación comercial, Austral y Lapa, ocurridos en la pasada década en nuestro país, dan claro testimonio de esta situación.

2. De acuerdo a las últimas estadísticas sobre empleo, esta cifra representa solamente al $50 \%$ de la población activa. Por consiguiente, las cifras de accidentes y trastornos de salud son, en realidad, mayores. Asimismo, es de señalar que, al primer trimestre de 2006, el sistema de riesgos alcanzaba a aproximadamente 6.400 .000 personas.

3. Este concepto hace alusión a los conocimientos que poseen los operadores respecto de la seguridad, construidos fundamentalmente por y para la acción, diferentes de los conocimientos “científico-técnicos" manejados por los profesionales de la seguridad en el trabajo.

\section{AUTOR}

\section{MÁRIO POY}

Universidad de Palermo - Facultad de Ciencias Sociales Geronimo Salguera, 1351 - 1177 Buenos Aires mpoy@cuidad.com.ar 